\title{
BCCN4
}

\section{FIVE-ELEMENT MODELS FOR LONG-TERM RESPONSE OF PGFRP MEMBERS}

\author{
$\underline{\text { Daniel C. T. Cardoso }}^{(1)}$ and Kent A. Harries ${ }^{(2,3)}$
}

(1) Department of Civil and Environmental Engineering, Pontifícia Universidade Católica do Rio de Janeiro, Brazil

(2) Department of Civil Engineering, University of Pittsburgh, United States

(3) Department of Architecture and Civil Engineering, University of Bath, United Kingdom

https://doi.org/10.21452/bccm4.2018.06.02

\begin{abstract}
Pultruded glass-fiber reinforced polymers (pGFRP) exhibit significant viscoelastic behavior even at room temperature, which may result in long-term loss of stability or excessive deflection. Several works in the literature have investigated creep of pGFRP, intending to characterize time-dependent behavior and to develop safe provisions for structural design of pGFRP members. The three-parameter Findley Power Law has been widely used by researchers and is usually calibrated to creep experiments conducted at constant stress and temperature. However, this empiricism and its lack of correlation with a mechanical model result in little predictive capacity for different resin types and fiber content. In this work, innovative linear viscoelastic models combining essentially elastic behavior of fiber and viscoelastic nature of matrix is proposed to represent the long-term behavior of pGFRP subject to stresses parallel or perpendicular to fiber direction. The model can also be used to assess long-term composite behavior for complex load history and for other viscoelastic phenomena such as recovery and relaxation, usually not addressed by Findley's law. The experimental results from a short and long-term mechanical characterization of a vinylesterbased pGFRP are reported and compared with the proposed approach, showing that the model satisfactorily predicts creep and recovery of such composites for a given time span. It is also shown that the presence of fibers parallel to load direction result in much lower relative compliance and quicker recovery.
\end{abstract}

\section{INTRODUCTION}

Pultruded glass-fiber reinforced polymers (pGFRP) are gaining acceptance in civil engineering applications particularly due to their high strength, lightweight and non-corrosive nature. However, some aspects of pGFRP material behavior are not fully understood, including long-term performance. The limited knowledge has led to conservative design considerations in current and in-development design standards, potentially increasing the cost of structures made with the pGFRP [1]. 
The pronounced creep behavior of pGFRP is usually attributed to the viscoelastic nature of the polymer resin, which is highly dependent on the curing and post-curing conditions [2, 3]. In the pultrusion process, pGFRP are pulled with speeds typically ranging from 300 to $500 \mathrm{~mm} / \mathrm{min}$ through 500 to $1500 \mathrm{~mm}$ long dies heated to temperatures between 100 and $150{ }^{\circ} \mathrm{C}$, This process is likely to result in incomplete cure [3].Glass fibers may also exhibit a viscoelastic response, but usual at relatively greater stress levels, typically beyond those anticipated in structural service.

Findley's three-parameter empirical power law, described in Eq. 1, has been widely used to describe primary and secondary creep of polymers with good accuracy over a wide range of time, $t[4]$.

$\frac{\varepsilon(t)}{\sigma_{0}}=J(t)=\frac{1}{E_{0}}+\frac{1}{E_{m}} t^{n}$

In which $\varepsilon(t)$ is the long-term strain, $\sigma_{0}$ is the constant applied stress, $J(t)$ is the creep compliance and $E_{0}, E_{m}$ and $n$ are stress-independent parameters. This equation is usually adequate for low stress levels and its popularity is based on its simplicity and excellent agreement with experiments.

In a seminal study, Bradley et al. [2] adopted flexural creep tests to characterize neat vinylester and polyester resins having different curing and post-curing conditions using Findley's power law. The authors observed that i) polyester-based specimens exhibited greater compliance than vinylester for the same curing condition; ii) temperature-cured specimens resulted in much lower creep exponent, $n$, than those cured at room temperatures; and iii) important reduction in creep compliances were observed with post-curing time. Differences are associated to the type of chemical structure of each resin and to the timetemperature relation for cross-linking completion. Finally, the authors tested vinylester specimens reinforced with woven E-glass fabric and showed that creep compliances are significantly reduced by the presence of fibers.

Findley's equation has also been successfully used by several authors for pGFRP, treating the composite as a single viscoelastic bulk material [1,5-7]. One of the drawbacks is the large dispersion of values for the parameters reported in literature, which is certainly associated to differences of resin properties and fiber volume ratios, $V_{f}$, in each study. For example, creep exponents ranging from $n=0.14$ to $n=0.36$ have been reported in literature for pGFRP [1]. It can be noted that parameters are also dependent on the loading type and direction. Harries et al. [1] and Sá et al. [7] summarized some previous works intending to investigate the longterm behavior of pGFRP, and it can be seen that most creep data have been obtained for tension, compression or flexure of specimens loaded parallel to the pultrusion direction (i.e., in the longitudinal direction). To date, limited data has been reported about shear creep [8] and, to the best of authors' knowledge, there are no works addressing specimens subject to stresses perpendicular to the pultrusion direction (transverse). These two cases correspond to conditions where behavior is usually strongly dependent on resin properties. Moreover, information regarding relaxation, recovery and behavior under complex loading history are very limited. Sá et al. [7], for example, reported large immediate recovery in full scale pGFRP beams.

Findley's law is empirical and its parameters are usually determined to fit experimental results obtained under conditions of constant stress. The lack of correlation with a mechanical model may result in little predictive capacity for a complex load history and for relevant 
phenomena for viscoelastic materials such as relaxation and immediate and delayed recovery. Linear models combining basic units - springs and dashpots - may be used along with superposition for full mathematical representation of general viscoelastic behavior. Usually, models using fewer basic units are easier for characterization and allow for better interpretation of mechanical behavior, but few viscoelastic materials have creep characteristics that can be described by such simple models. On the other hand, these simple models can be used to study long term behavior over a given time span [4]. Sá et al. [9], for example, reported good agreement with experimental creep results when using DirichletProny or Burgers-Kelvin models, whereas a poorer agreement was reported for simpler fourelement Burgers' model. A Burgers' model for FRP laminates starting from properties of single phases was also proposed by Ascione et al. [10] and validated with experiments.

The present study aims to propose simple five-element models combining fiber and matrix individual mechanical properties, in a viscoelastic extension of the so-called rule-of-mixtures (RoM). The proposed approach accounts for different matrix properties and fiber contents, as well as for loading direction. The influence of fiber architecture on long-term behavior is discussed. Creep, recovery and relaxation phenomena are studied and contributions of matrix and fiber to behavior are discussed for loading parallel and perpendicular to the pultrusion direction. The partial results from an ongoing experimental program are compared to those predicted using the proposed method.

\section{MECHANICAL MODELS}

The proposed mechanical models are based on the following assumptions: i) fibers can be described by a linear elastic model, with negligible viscous deformation, as reported by Ascione et al. [10]; ii) the matrix is represented by a Burgers' viscoelastic model; iii) fibers are unidirectional and homogeneously distributed throughout matrix; and iv) perfect adhesion between constituents is assumed. In Figures $1 \mathrm{a}$ and $1 \mathrm{~b}$, models for loading applied parallel and perpendicular to the fiber direction are presented, respectively. In the former case, applied force is shared between reinforcement and matrix and the same strain is experienced by both constituents (association in parallel); for the latter case, applied force is the same for both reinforcement and matrix and the final strain is the sum of the strains obtained for each constituent (association in series). Similarly to the rule-of-mixtures, the properties for springs and dashpots $\left(k_{i}\right.$ and $\eta_{i}$ ) for each model are defined according to constituent properties and their corresponding volume fraction, as follows:

- load parallel to fiber direction (Fig. 1a): stiffnesses are obtained multiplying constituent stiffnesses by their corresponding volume fraction (e.g. $k_{f}=E_{f} V_{f}$, where $E_{f}$ is the fiber modulus; $\eta_{1}=c_{1} V_{m}$ where $c_{1}$ is the matrix primary dashpot and $V_{m}=1-V_{f}$ is the matrix content in volume);

- load perpendicular to fiber direction (Fig. 1b): stiffnesses are obtained dividing constituent stiffnesses by their corresponding volume fraction (e.g. $k_{f}=E_{f} / V_{f}$ and $\eta_{1}=c_{1} / V_{m}$ ); 


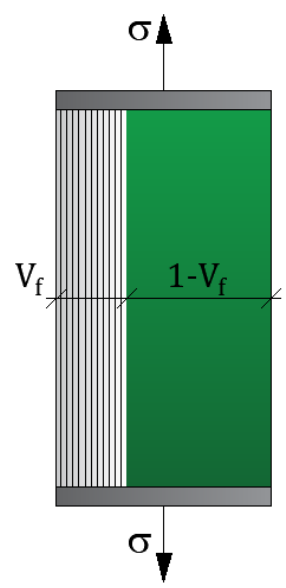

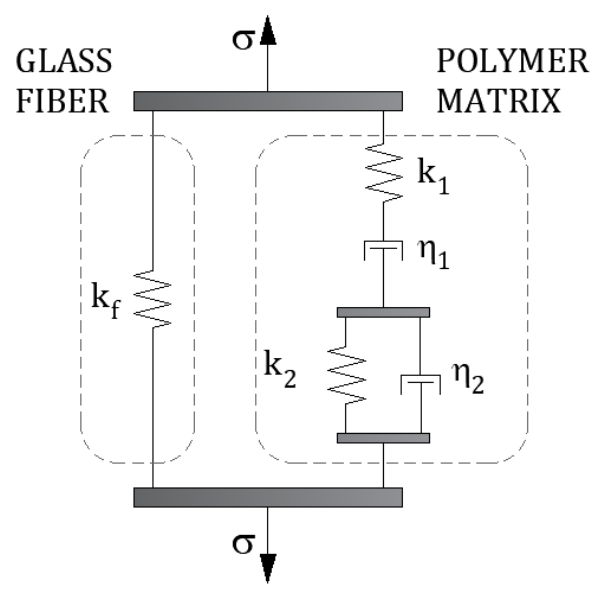

(a)

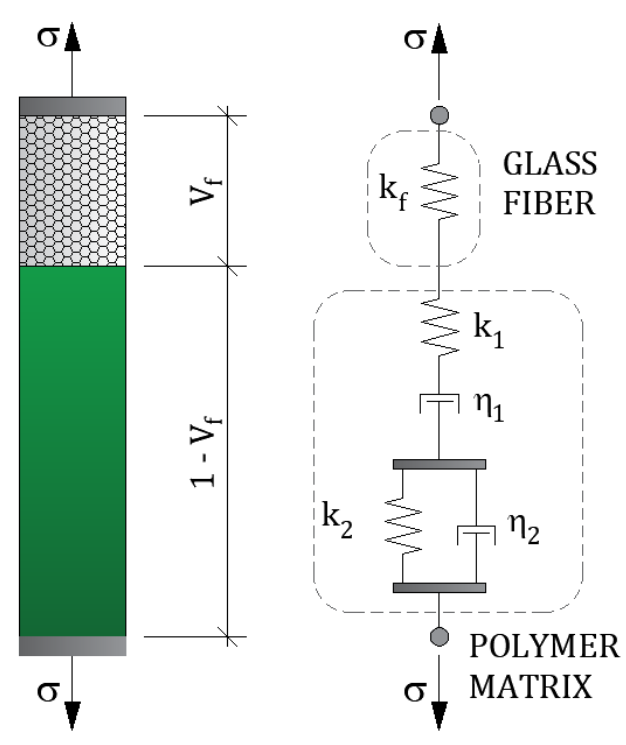

(b)

Figure 1 : Proposed mechanical models: a) load parallel to fiber direction (association in parallel); and b) load perpendicular to fiber direction (association in series).

In Figure 2a, relative creep compliances obtained for specimens loaded parallel to the fiber direction are presented for different fiber contents $\left(V_{f}\right)$. Graphs in Figure 2 were obtained considering vinylester resin post-cured at $93{ }^{\circ} \mathrm{C}$ for one week, as given in Table 1 [2]. For $V_{f}=0$, the proposed model reduces to Burgers' model and a good agreement is obtained with experimental data for the time span considered. For $V_{f}>0$, significant reductions in compliance are predicted. In general, as the matrix dashpots 'release strain', the overall matrix stiffness falls, affecting the stress distribution between matrix and fiber: the load carried by the fibers increases and the force transferred through the matrix decreases. This change in load distribution also leads to a reduction in the strain mobilized in matrix spring $k_{1}$.

Table 1 : Burgers’ model parameters for vinylester with different post-curing conditions [2].

\begin{tabular}{l|cc}
\multirow{2}{*}{ Property } & \multicolumn{2}{|c}{ Post-curing condition } \\
& RT - 1 month & $\mathbf{9 3}{ }^{\mathbf{0}} \mathbf{C} \mathbf{- 1}$ week \\
\hline Primary modulus of vinylester resin $\left(\boldsymbol{E}_{\mathbf{1}}\right)$ & $3.5 \mathrm{GPa}^{*}$ & $3.5 \mathrm{GPa}^{*}$ \\
Secondary modulus of vinylester resin $\left(\boldsymbol{E}_{2}\right)$ & $9.0 \mathrm{GPa}$ & $17 \mathrm{GPa}$ \\
Primary dashpot of vinylester resin $\left(\boldsymbol{c}_{\boldsymbol{1}}\right)$ & $1.0 \times 10^{6} \mathrm{GPa} . \mathrm{s}$ & $4.0 \times 10^{6} \mathrm{GPa} . \mathrm{s}$ \\
Secondary dashpot of vinylester resin $\left(\boldsymbol{c}_{\boldsymbol{2}}\right)$ & $5.0 \times 10^{4} \mathrm{GPa} . \mathrm{s}$ & $7.5 \times 10^{4} \mathrm{GPa} . \mathrm{s}$ \\
* average estimated & & \\
RT $=$ room temperature & &
\end{tabular}

When loaded in the perpendicular to the fiber direction, in-series springs $k_{f}$ and $k_{1}$ are subject to the same force. Therefore, they can be treated as a single equivalent spring with $k_{e q}$ $=k_{f} k_{1} /\left(k_{f}+k_{1}\right)$ and the model reduces to a simple Burgers' model. As can be seen in Figure $2 \mathrm{~b}$, for loading perpendicular, relative creep compliances decrease with increasing fiber contents, but to a much significant degree as compared to the case where fibers are parallel to load 
direction (Figure 2a). The proposed models can also be used to predict relaxation and recovery phenomena and the strong influence of fiber content and loading direction can be observed. A much quicker recovery and reduced relaxation is predicted when loads are applied parallel to fiber direction.

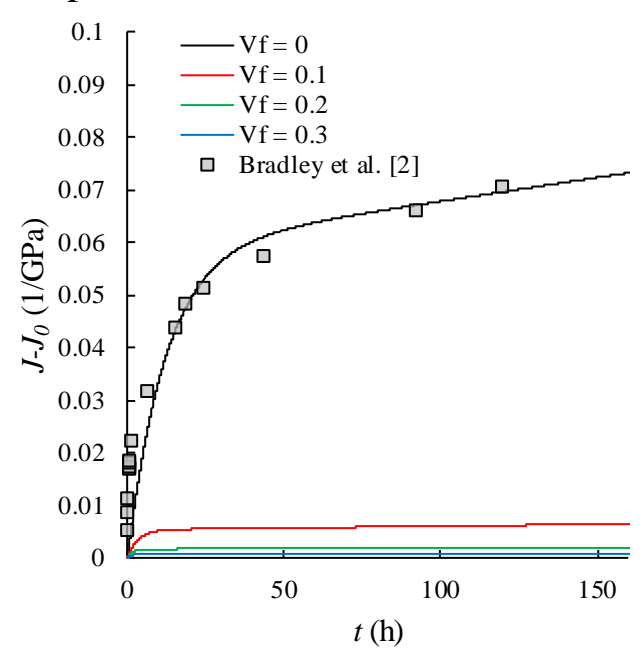

(a)

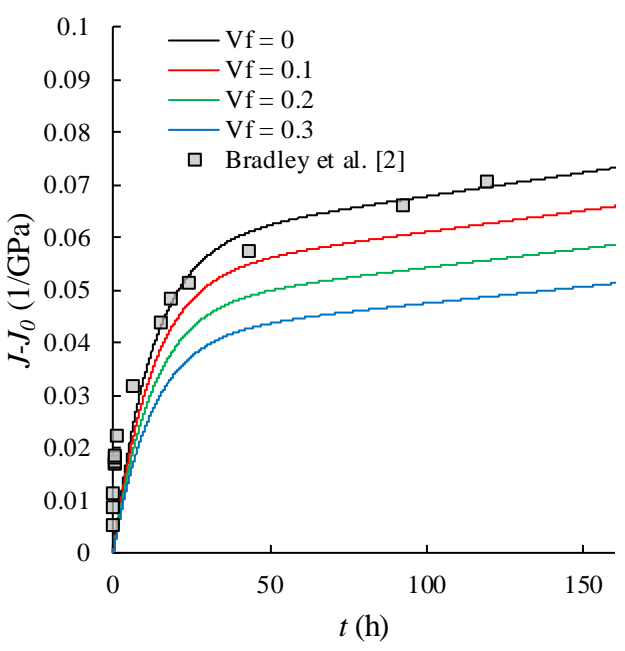

(b)

Figure 2 : Predicted creep behavior for members with different fiber contents: a) loads applied parallel to fiber direction; b) loads applied perpendicular to fiber direction.

In real pGFRP, fiber volume content ranges from $V_{f}=0.20$ to $V_{f}=0.50$. This volume is composed of i) continuous strand mat (CSM), comprising about $10-20 \%$ of $V_{f}$, (thicker pultruded plates have lower proportions of CSM); and ii) roving layers, comprising the remaining 80 to $90 \%$ of $V_{f}$. Surface veils made of polyester are also used at the outer faces. Whereas roving layers are orthotropic due to the unidirectional contribution of fibers in the direction of pultrusion, CSM layers are usually assumed to be isotropic due to approximately equal contribution of randomly oriented fibers in both directions [11]. In Figure 3a, an idealized cross-section of thickness $h$ is presented and, in Figure 3b, the corresponding distributions of flexural strains and stresses are shown, from which it can be highlighted that each layer contributes differently to resisting the applied moment, as in a laminate. Therefore, the effectiveness of fibers is reduced with respect to the case of pure axial load and is strongly dependent on the fiber architecture [12]. In the present work, for simplicity, the concept of effective volume fraction, $V_{f, e f f}$, is introduced to represent the fiber content parallel to the loading direction homogeneously distributed throughout matrix for a linear stress distribution. Finally, it can be noted that, due to the presence of CSM in typical pGFRP, there will be always a certain proportion of fibers parallel to the direction of load. The presence of fillers may also affect the behavior, but is not considered in the present study.

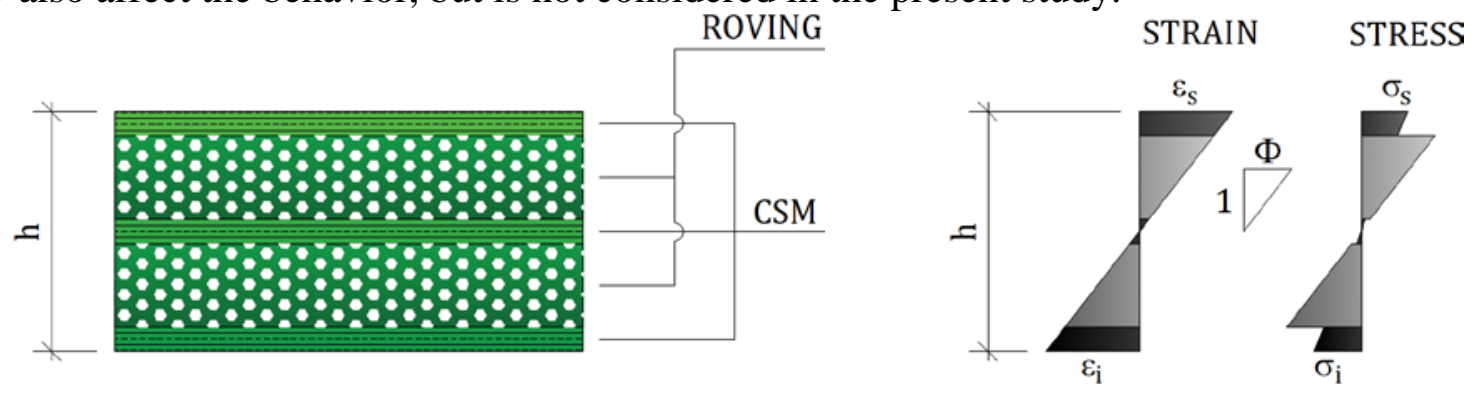


Figure 3 : Behavior of idealized pGFRP: a) fiber architecture; b) flexural strain and stress.

\section{EXPERIMENTAL PROGRAM}

The experimental program consisted of short and long-term flexural characterization of a pGFRP composite plates loaded both parallel and perpendicular to the pultrusion direction. Specimens used in this study were extracted from a 152x6.3 mm vinylester-based pGFRP plates, an example of which is shown in Figure 4a. According to the manufacturer, the plates were produced using Dion ${ }^{\circledR}$ Impact 9102 GP resin or equivalent and E-glass fibers. The plates were fabricated with a pull speed of $280 \mathrm{~mm} / \mathrm{min}$ through a $500 \mathrm{~mm}$ long mold having a temperature of approximately $150^{\circ} \mathrm{C}$.

\subsection{Fiber Content and Architecture}

To determine the fiber content, burnout tests were carried out according to ASTM D3171. A fiber volume fraction of approximately $V_{f}=0.30$ was calculated; $15 \%$ of this volume corresponded to CSM. An optical microscope was also used to analyze the typical fiber architecture, which was found to be quite uniform as seen in Figure 4b.

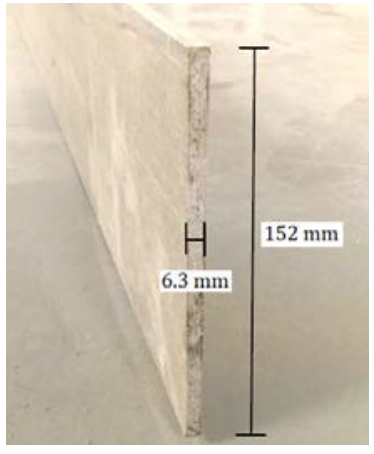

(a)

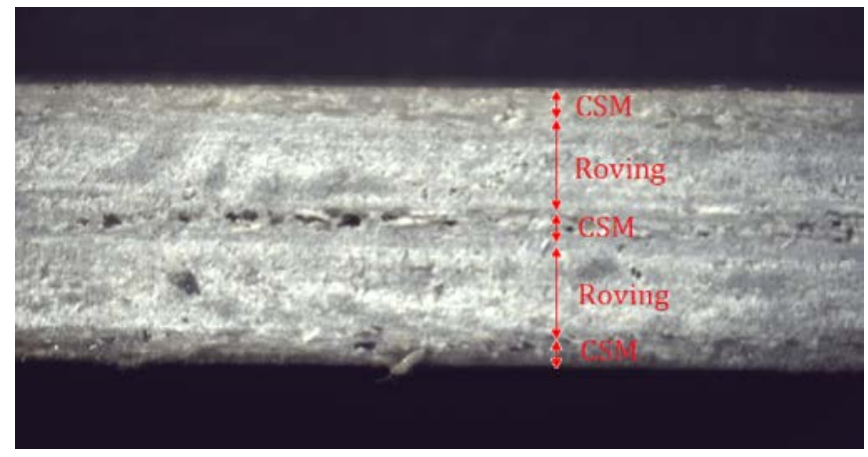

(b)

Figure 4 : pGFRP material studied: a) original plate; b) fiber architecture.

\subsection{Short and Long-Term Mechanical Characterization}

To obtain short and long-term mechanical properties in the longitudinal direction, three 30x300 mm specimens were extracted from the plates and tested in a four-point bending configuration with total and shear spans of 250 and $100 \mathrm{~mm}$, respectively (Figure 5a). It is important to note that the shear span-to-depth ratio exceeds 10, resulting in negligible contribution of shear deformation to the total deflection. A displacement transducer was used at the top of the rigid loading beam and strain gages were positioned on both top and bottom faces of the specimens at the constant moment region, as shown in Figure 5a. For short-term characterization, loads were applied in increments of $50 \mathrm{~N}$ up to $250 \mathrm{~N}$ (free weights), in less than one minute, and the unloaded in the same way. The procedure was repeated three times and a consistency in the results was observed. Following this initial loading, specimens were unloaded and remained in this condition at least for one week before being reloaded using similar increments for the long-term testing sequence. For long-term characterization, specimens were loaded with 200 or $250 \mathrm{~N}$ (see Table 2) for approximately $170 \mathrm{~h}$, when they were unloaded. Recovery data were recorded for an additional $50 \mathrm{~h}$ period of time.

For the transverse direction, three 25x152 mm specimens were used and tested in threepoint bending over a $140 \mathrm{~mm}$ span (Figure 5b). A displacement transducer was used at mid- 
span. The same procedure - although with different applied loads - as described for longitudinal specimens was used. For short-term characterization, loads were applied in $10 \mathrm{~N}$ increments up to $80 \mathrm{~N}$ and for long-term characterization, specimens were loaded with 50 or $80 \mathrm{~N}$ (see Table 2).
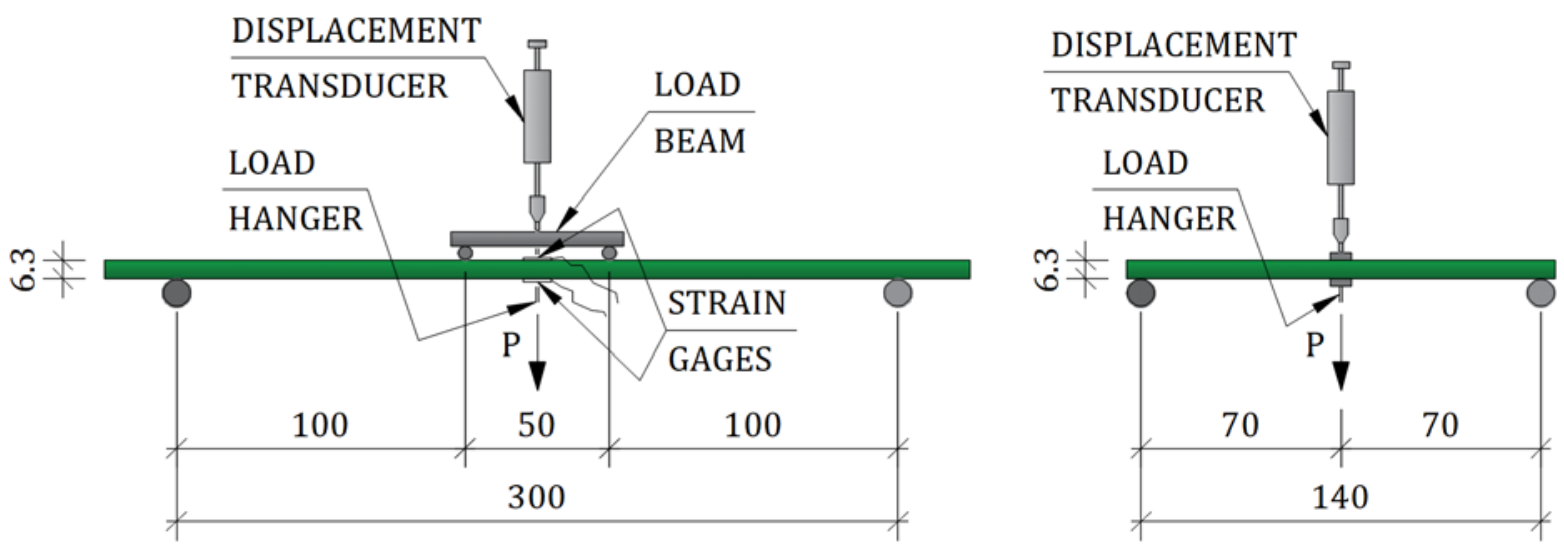

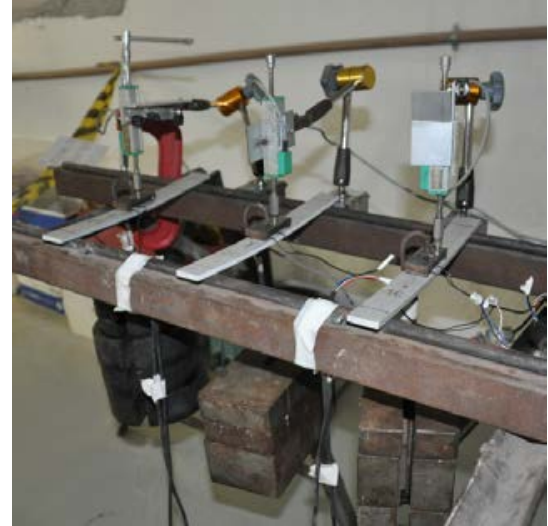

(a)

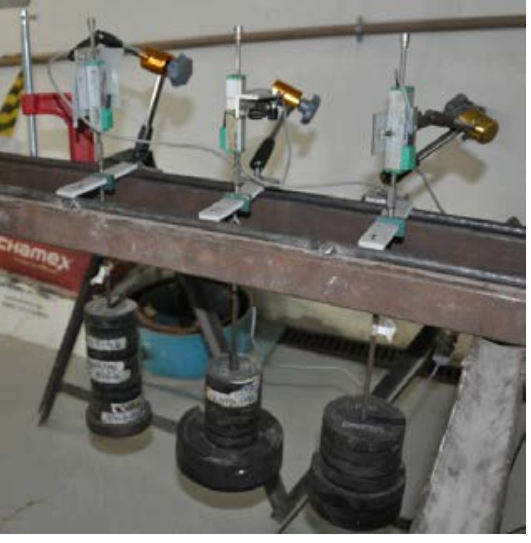

(b)

Figure 5 : Scheme and view of characterization tests: a) longitudinal direction; b) transverse direction (dimensions in $\mathrm{mm}$ ).

\section{DISCUSSION OF RESULTS}

In Table 2, measured dimensions (width $b$ and thickness $h$ ) of specimens are reported, as well as the elastic flexural moduli obtained from short-term characterization, $E_{b}$, and effective fiber volume fractions. $V_{f, \text { eff }}$ was back-calculated from the rule-of-mixtures, assuming moduli of elasticity of fiber and matrix $E_{f}=72 \mathrm{GPa}$ and $E_{1}=3.5 \mathrm{GPa}$, respectively.

Table 2 :Measured dimensions, elastic moduli and effective volume fraction.

\begin{tabular}{c|ccccc} 
Specimen & $\mathbf{b}(\mathbf{m m})$ & $\mathbf{h}(\mathbf{m m})$ & $\boldsymbol{E}_{\boldsymbol{b}}(\mathbf{G P a})$ & $\boldsymbol{V}_{f, e f f}$ & $\begin{array}{c}\text { Long-term } \\
\text { load (N) }\end{array}$ \\
\hline 1 Longitudinal & 30.2 & 6.12 & 21.9 & 0.27 & 250 \\
2 Longitudinal & 28.8 & 6.15 & 23.4 & 0.29 & 200 \\
3 Longitudinal & 31.4 & 6.10 & 22.6 & 0.28 & 200
\end{tabular}


$4^{\text {th }}$ Brazilian Conference on Composite Materials. Rio de Janeiro, July 22 $2^{\text {nd }}-25^{\text {th }}, 2018$

\begin{tabular}{l|lllll} 
1 Transverse & 25.0 & 6.12 & 5.6 & 0.01 & 80 \\
2 Transverse & 23.2 & 6.18 & 5.5 & 0.01 & 80 \\
3 Transverse & 23.7 & 6.14 & 5.6 & 0.01 & 50
\end{tabular}

In Figure 6a, creep and recovery compliances for longitudinal specimens are presented. Results are relatively consistent for the three specimens, with differences only in initial compliance (associated to initial modulus). A large elastic recovery can be observed for the specimens. In Figure 6b, relative creep compliance obtained experimentally are presented. Results obtained with the proposed model using resin properties for different post-curing conditions given in Table 1: room temperature (RT) for 1 month and $93^{\circ} \mathrm{C}$ for 1 week (crosslinking completion). An important difference between the model and experiments was observed immediately following loading, with the model predicting an initially greater creep rate. It can be seen that actual behavior corresponds to an intermediate case, but can satisfactorily be represented by the proposed model considering vinylester properties for $93^{\circ} \mathrm{C}$ post-curing. In Figures $6 \mathrm{c}$ and 6d, similar graphs are presented for the specimens loaded in the transverse direction. Much greater compliances and relative compliances can be observed with respect to the longitudinal direction, as well as a less significant (greater residual compliance) recovery. The proposed model once again showed good agreement with experiments. Similar conclusions were obtained with strain gage data from the longitudinal specimens.

\section{CONCLUSION}

In this work, innovative mechanical models intending to represent orthotropic pGFRP composite viscoelastic behavior was proposed. The proposed models account for fiber content and for the properties of each phase of the composite and can be used to predict long-term behavior of such composites under complex loading history. Predictions obtained using the proposed models showed satisfactory agreement with creep-recovery experiments performed for longitudinal and transverse specimens. It is demonstrated that behavior is strongly affected by the presence of fibers parallel to the load direction, reducing relative compliance and promoting a greater elastic recovery. Mechanical characterization of glass fibers and resin for both short and long-term behavior are underway, as well as relaxation and shear creeprecovery tests.
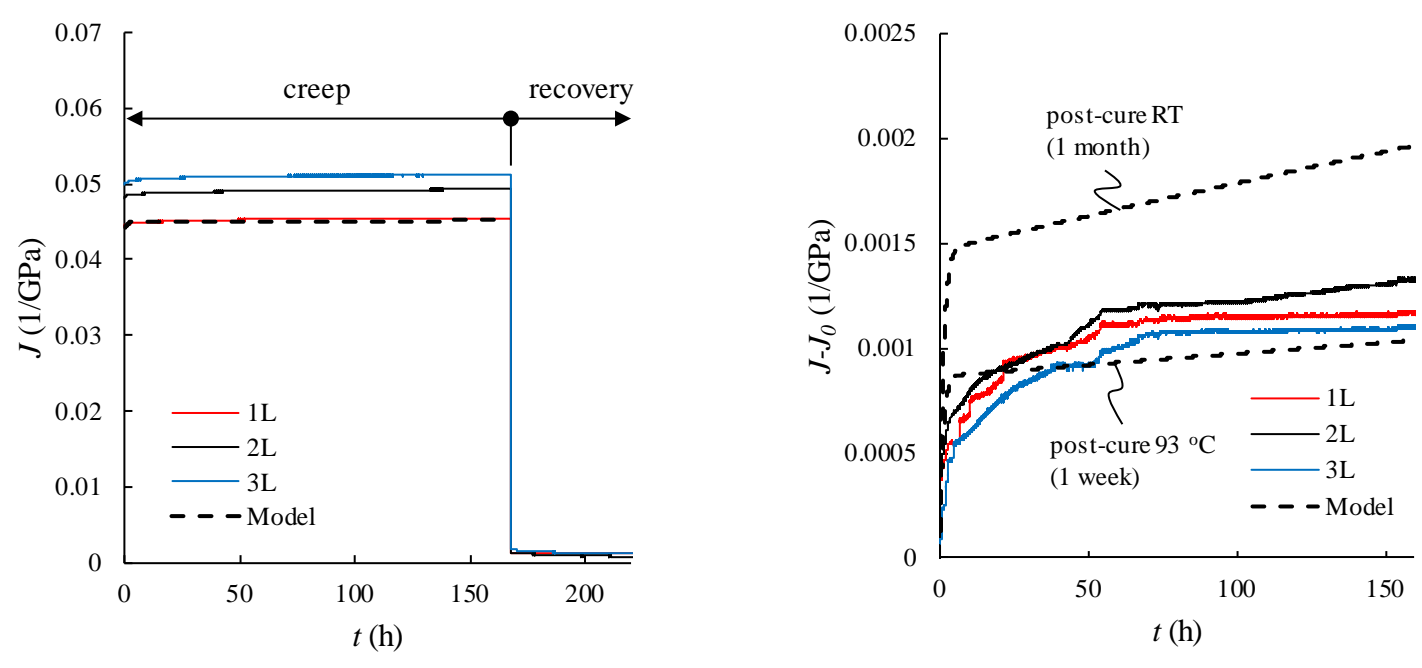
(a)

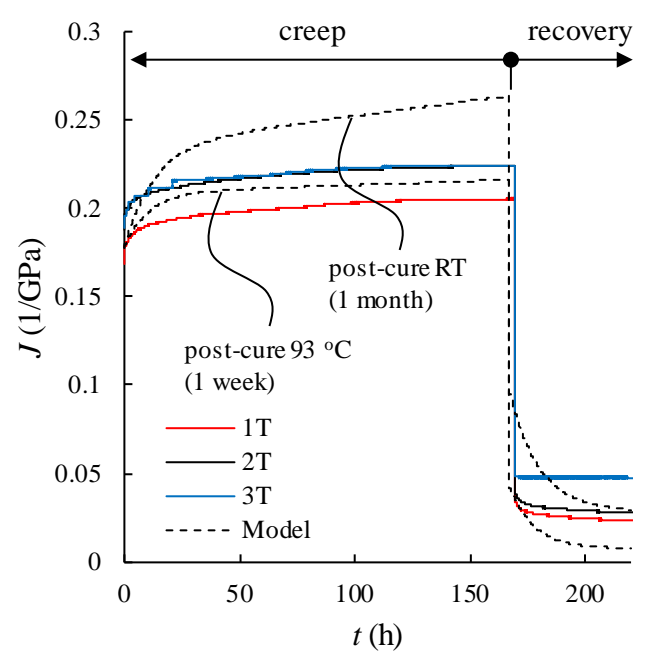

(c) (b)

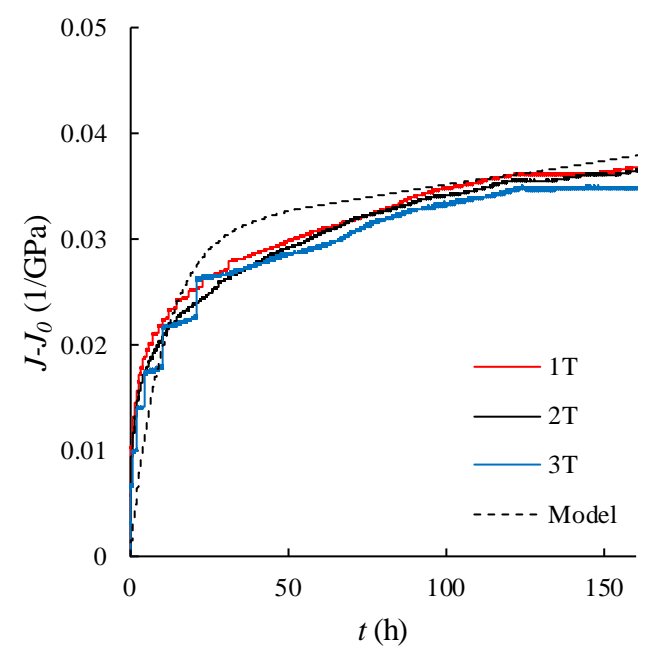

(d)

Figure 6 : Creep and recovery results: a) creep and recovery (longitudinal); b) relative compliance (longitudinal); c) creep and recovery (transverse); d) relative compliance

(transverse);

\section{ACKNOWLEDGEMENTS}

The authors would like to acknowledge Cogumelo for supplying the material used in this research. The first author acknowledges $\mathrm{CNPq}$ for financial support through process 402026/2016-0.

\section{REFERENCES}

[1] Harries, K. A., Guo, Q., \& Cardoso, D. (2017). Creep and creep buckling of pultruded glassreinforced polymer members. Composite Structures, 181, 315-324.

[2] Bradley, S. W., Puckett, P. M., Bradley, W. L., \& Sue, H. J. (1998). Viscoelastic creep characteristics of neat thermosets and thermosets reinforced with E-glass. Journal of Composites, Technology and Research, 20(1), 51-58.

[3] Batch, G. L., \& Macosko, C. W. (1993). Heat transfer and cure in pultrusion: model and experimental verification. AIChE Journal, 39(7), 1228-1241.

[4] Findley, W. N., Lai, J. S. \& Onaran, K. (1976). 'Creep and relaxation on nonlinear viscoelastic materials'. Dover Publications.

[5] Bank, L. C., \& Mosallam, A. S. (1992). Creep and failure of a full-size fiber-reinforced plastic pultruded frame. Composites Engineering, 2(3), 213-227.

[6] Scott, D. W., \& Zureick, A. H. (1998). Compression creep of a pultruded E-glass/vinylester composite. Composites Science and Technology, 58(8), 1361-1369.

[7] Sá, M. F., Gomes, A. M., Correia, J. R., \& Silvestre, N. (2011). Creep behavior of pultruded GFRP elements-Part 1: Literature review and experimental study. Composite Structures, 93(10), 2450-2459.

[8] Bottoni, M., Mazzotti, C., \& Savoia, M. (2014). Creep tests on GFRP pultruded specimens subjected to traction or shear. Composite Structures, 108, 514-523.

[9] Sá, M. F., Gomes, A. M., Correia, J. R., \& Silvestre, N. (2011). Creep behavior of pultruded GFRP elements-Part 2: Analytical study. Composite structures, 93(9), 2409-2418.

[10] Ascione, L., Berardi, V. P., \& D’Aponte, A. (2012). Creep phenomena in FRP materials. Mechanics Research Communications, 43, 15-21. 
$4^{\text {th }}$ Brazilian Conference on Composite Materials. Rio de Janeiro, July $22^{\text {nd }}-25^{\text {th }}$, 2018

[11] Naughton, B. P., Panhuizen, F., \& Vermeulen, A. C. (1985). The elastic properties of chopped strand mat and woven roving in GR laminae. Journal of reinforced plastics and composites, 4(2), 195-204.

[12] Liu, T., Harries, K.A. and Guo, Q. (2018) Effects of Fiber Architecture on Flexure Properties of Pultruded GFRP Plates and Sections, Proceedings of the $9^{\text {th }}$ International Conference on Fibre-Reinforced Polymer Composites in Construction (CICE 2018), Paris, July 2018. 